\title{
Modelling the cost-effectiveness of pharmacotherapy compared to CBT and combination therapy for the treatment of moderate to severe depression in the UK
}

\author{
L. Koeser ${ }^{*}$, V. Donisi ${ }^{2}$, D.P. Goldberg ${ }^{1}$, P. McCrone ${ }^{1}$ \\ 1 Institute of Psychiatry, King's College London, UK \\ 2 Department of Public Health and Community Medicine, Section of Psychiatry, \\ University of Verona, Italy
}

\section{Background.}

The National Institute of Health and Care Excellence (NICE) in England and Wales recommends the combination of pharmacotherapy and psychotherapy for the treatment of moderate to severe depression. However, the cost-effectiveness analysis on which these recommendations are based have not included psychotherapy as monotherapy as a potential option. For this reason, we aimed to update, augment and refine the existing economic evaluation.

\section{Methods.}

We constructed a decision analytic model with a 27-month time horizon. We compared pharmacotherapy with cognitive behavioural therapy (CBT) and combination treatment for moderate to severe depression in secondary care from a healthcare service perspective. We reviewed the literature to identify relevant evidence and, where possible, synthesised evidence from clinical trials in a meta-analysis to inform model parameters.

\section{Results.}

The model suggested that CBT as monotherapy was most likely to be the most costeffective treatment option a cost per QALY threshold above $£ 22,000$. It dominated combination treatment and had an incremental cost-effectiveness ratio of $£ 20,039$ per quality-adjusted life year compared to pharmacotherapy. There was significant decision uncertainty in the probabilistic and deterministic sensitivity analyses.

\section{Conclusions.}

Contrary to previous NICE guidance, the results indicated that even for those patients for whom pharmacotherapy is acceptable, CBT as monotherapy may be a cost-effective treatment option. However, this conclusion was based on a limited evidence base, particularly for combination treatment. In addition, this evidence cannot easily be transferred to a primary care setting. 


\section{Financial Support}

This research received no specific grant from any funding agency, commercial or not-forprofit sectors.

* Author for correspondence:

Health Service and Population Research Department Institute of Psychiatry

King's College London

Box P024, De Crespigny Park

London SE5 8AF, UK

e-mail: leonardo.koeser@kcl.ac.uk 


\section{Introduction}

Depression is the most common psychiatric disorder. In 2007, 1.24 million people were estimated to suffer from this condition in England, which resulted in health and social care costs of $£ 1.7$ billion. By 2026, the number of people and costs associated with depression are projected to rise to 1.45 million and £3 billion, respectively (McCrone et al., 2008). Treatment for depression in the England and Wales is guided by a stepped-care model (NICE, 2009). Less intrusive and costly treatments such as guided self-help, physical exercise or computerised cognitive behavioural therapy are recommended for patients with sub-threshold or mild symptoms. Access to more intensive treatments (i.e. pharmacotherapy, psychotherapy and combination therapy) is indicated for patients who do not respond to or decline these options, or those presenting with more severe types of depression. In order to make best use of limited health care budgets, the evaluation of the relative cost-effectiveness of these three treatment alternatives has been a chief concern in the UK. Many studies assessing different types of antidepressants exist but comparisons between difficult treatment classes are less common. The fundamental question in this context is whether the added costs of psychotherapy, singly or in combination with pharmacotherapy, due to the more extensive contact with clinicians is outweighed by its potential benefits over pharmacotherapy.

Current guidance by the National Institute for Health and Care Excellence (NICE) recommends the provision of a combination of pharmacotherapy and cognitive behavioural therapy (CBT) or Interpersonal psychotherapy (IPT) for both moderate and severe depression (NICE, 2009). However, this recommendation is based on an economic analysis that does not include psychotherapy in form of a monotherapy as a treatment alternative. The NICE (2009) guidance development group (GDG) justified this exclusion by noting that the "clinical evidence review showed no overall superiority for CBT alone on treatment outcomes" and that it had significantly higher treatment costs. Yet, elsewhere the GDG states that "it was not possible to identify a benefit of adding antidepressants to CBT", and that "CBT alone was found to be better than antidepressant alone when compared with combined treatment". In addition, in practice many patients in the UK do not appear to receive recommended treatments because of supply constraints in the provision of psychotherapy (Gyani et al., 2012). For these reasons, this study aimed to update and refine the available evidence on the comparative cost-effectiveness of treatments for depression to inform decision making in a secondary care setting. In particular, besides comparing pharmacotherapy and combination treatment we also included CBT monotherapy in the economic evaluation. 


\section{Methods}

\section{Patient population and comparators}

The target population of our decision analytic model were adults with moderate or severe major depressive disorder (MDD) according to cut-off scores on two common depression rating scales: the Hamilton Depression Scale (HAMD-17) (scores $\geq 14$ ) and the Beck Depression Inventory (BDI) (scores $\geq 17$ ) (American Psychiatric Association, 2000). Given the amount of available data, we chose to combine the available evidence base using a decision tree model. We included studies in which any antidepressant medication, face-to-face CBT or cognitive therapy and/or combination treatment were compared. We excluded other types of psychotherapies as they are not commonly available in the UK National Health Service and because to date less empirical evidence on them is available. We did not consider other forms of delivering psychotherapy such as group therapy or computerised CBT. We modelled treatment in a secondary care setting because the vast majority of patients in eligible studies recruited patients in this context. We only considered first line treatment and did not allow for treatment augmentation or switching during the acute treatment phase.

\section{Model structure}

None of the clinical trials relevant for our model followed up patients for more than 24 months after the end of the acute treatment phase. Therefore, we compared the costeffectiveness of antidepressants, CBT and combination therapy over a 27-month time horizon consisting of 3-month acute treatment phase, a follow-up at 12 months and 24 months after the end of acute treatment. We distinguished between three post-treatment clinical states or events: remission (or full response), response (or partial remission) and non-response.

Premature termination of treatment is common in depression. In the model, patients were assumed to discontinue treatment because of positive reasons (i.e. remission or the perception thereof) or negative ones (i.e. no improvements in symptoms and/or side effects). Patients who remitted or responded to treatment were thought to be at risk of relapse in the follow-up phases of the model. Figure 1 shows the possible transition pathways of the model. 


\section{Event probabilities}

Where possible, we obtained data for model parameters from randomised controlled trials (RCTs) because they are believed to minimise the risk of bias (NICE, 2009). A regularly updated database by Cuijpers et al. (2008) contains a catalogue of RCTs that compare the effects of psychological treatments (both singly and in combination) in adults with depressive disorders with a control intervention. VD and LK independently screened the January 2013 version of this database to identify relevant head-to-head comparisons. We included English language studies only.

We extracted data on remission, response and dropout rates if the study reported these outcomes at completion of the acute treatment phase. We considered patients with a score of 7 or less on the HAMD-17 to be in remission, those reaching a score between 8 and 13 to be responders and those with a score of 14 or above as non-responders (NICE, 2009). However, we allowed for $a \pm 1$ point margin in the cut-off definitions due to slight variations between studies. We extracted the data on an intention to treat basis. For consistency with NICE (2009) guidelines, we only extracted data on relapse rates from trials that incorporated some form of maintenance treatment, i.e. pharmacotherapy had to be continued beyond the end of the acute phase and 'booster' sessions had to available to patients both in the monotherapy and combination therapy arms. In light of the small number of studies that met this criterion, we allowed for any definition of relapse and allowed for studies that included some patients that may have been responders but not remitters according to our definition. In practice, in all included trials pharmacotherapy was discontinued at 6 or 12 month after remission. Based on our knowledge of the disease area and for sake of clarity, we considered it to be most appropriate to take a 'worst-case scenario' approach to missing data (Higgins and Green, 2008). In other words, we assumed that patients with a missing endpoint assessment in the acute and follow-up phase would be in the least favourable health status (i.e. non-response or relapse) and if it was unclear at what point patient dropped out of the study during the post-acute follow-up, we assumed that this occurred before the first-follow up. There appeared to be some ambiguities in how the data was reported in the follow-up studies. Given the importance of relapse rates for the results of the model, we individually comment on our approach to relapse rate data extraction in Appendix C.

We were unable to identify randomised trials that compared relapse rates among patients responding to treatment in isolation from remitters according to our definition of these subgroups. For this reason, over the first twelve months of follow-up, for the 
pharmacotherapy arm in our model we used the relapse rate of patients with a HAMD-17 score between 8 and 13 in the trial by Kuyken et al. (2008) who were treated with antidepressants. Conditional on not having relapsed until this point, we assumed that the relapse rate among pharmacotherapy treatment responders over the second 12 month follow-up was the same as among remitters. It appeared plausible that the direction of relative differences in relapse rates between treatments in terms of protecting against relapse would be the same among patients who were in remission after the acute treatment phase as for those responding to treatment. In absence of any direct evidence to support this belief or data on the relative magnitude of effects, we adopted a conservative approach aimed to reflect this notion in our model without unduly favouring any of the three interventions. Specifically, we assumed that the risk differences in relapse rates between the three interventions would be the same as among remitters but multiplied by a discount factor with mean 0.5 and standard deviation 0.3 , i.e. reduced by $50 \%$ on average.

A priori, there were clinical reasons to believe that some heterogeneity in observed treatment effects would be present. Besides the fact that different types of antidepressants were included in our comparisons, there is considerable variability in how both psychotherapy and pharmacotherapy are implemented in trials. Therefore, we synthesised the evidence using random-effects meta-analyses which does not assume the presence of a common effect across all studies (Borenstein et al., 2009, Riley et al., 2011). We adapted a Bayesian network meta-analysis framework proposed by Dias et al. (2013a) which accounts both direct and indirect treatment effects and correlations between arms within a trial. To better reflect the uncertainty resulting from heterogeneity between trials in a decision making context, we used predictive rather posterior distributions for baseline rates and treatment effects in our model (Dias et al., 2013b, Dias et al., 2013c). In other words, we modelled the uncertainty surrounding a hypothetical future 'roll out' of the interventions given the betweenstudy variance rather than the uncertainty surrounding the average presumed underlying treatment effects. We applied vague prior distributions for baseline and weakly informative $t$ family priors to treatment effects (Gelman et al., 2008). For between-study variances, on the other hand, we used an informative prior distributions based on a review by Turner et al. (2012) to stabilize our estimates. Put differently, when there were few studies available to inform the estimate of between study variance, such as in the follow-up phase, we assumed that variance between studies in our meta-analysis would be relatively similar to that in comparable meta-analyses in the literature rather than relying on the limited amount of existing data, whereas we allowed the data to 'dominate' when sufficient evidence was available. We used WinBUGS to run our analyses. The code which allows the replication of the entire decision model including these meta-analyses can be found in Appendix D. 
The literature contained little systematic evidence on the disease course of patients who discontinued depression treatment. Data by Radhakrishnan et al. (2013) as well as expert opinion elicited by NICE (2009) and Sado et al. (2009) suggested that approximately $20 \%$ of patients discontinue treatment due to recovery and this figure was used. We assumed that, regardless of initial treatment assignment, relapse rates at 12 month follow-up among patients who discontinued treatment because of feeling cured was equal to those of patients treated with a placebo during the acute phase in a study by Jarrett et al. (2000). In addition, we assumed that patients in this subgroup who which did not relapse of during the first 12 month follow-up were not at risk of relapse over the remaining time horizon of the model.

\section{Health-related quality of life}

We quantified the health benefits of the interventions using quality adjusted life years (QALYs). This approach assigns a preference-based weight, usually between 1 (representing full health) and 0 (representing death) to health states in an attempt to quantify the relative value of quality of life therein. This value is multiplied by the length of time spent in that health state to yield QALYs (Malek, 2000). The EuroQol 5 Dimensions (EQ-5D) is the instrument currently preferred by NICE to derive the preference weights for health states in adults (NICE, 2013). However, a review of the literature suggested that no published evidence was available mapping EQ-5D scores by depression status as defined by the HAMD-17 in this model. Therefore, we calculated mean EQ-5D utilities for remitters, responders and non-responders as defined above ourselves using data from a trial by Kuyken et al. (2008) on patients with recurrent depression. To account for the fact that repeated measures were available for most patients, we used a pooled ordinary least squares model with cluster robust standard errors. We assigned the same quality of life to patients dropping out of treatment due to side effects or no response as to those who completed the treatment but who did not respond.

\section{Costs}

We measured costs from a UK health care perspective and used a price year of 2012. Due to lack of robust empirical evidence, we based our costing for the interventions on a assumptions made in a previous cost-effectiveness analysis by Simon et al. (2006) using unit cost data from Curtis (2012). Since it was the most widely prescribed antidepressant in England in 2010, pharmacotherapy was assumed to consist of a $20 \mathrm{mg}$ daily dose of citalopram over a for a total of 15 months (llyas and Moncrieff, 2012). This is longer than 
treatment period than suggested by NICE (2009) guidance but consistent with the RCTs informing our model. As part of patient monitoring beyond what would be expected in usual care, patients treated with antidepressants were initially assumed to have two appointments with a psychiatric consultant and two with a specialist registrar each lasting 50 minutes. (NICE, 2009). CBT treatment was assumed to consist of 16 sessions during the acute treatment phase and two additional 'booster' sessions after that. We assumed that patients who discontinued pharmacotherapy dropped out of treatment after one month of treatment and one appointment with a psychiatric consultant whereas patients receiving CBT were assumed to drop out after four sessions. The cost for combination therapy in our model was the sum of the cost of pharmacotherapy and psychotherapy. We assumed that patients who did not respond to the treatment administered in the acute phase would not receive any booster CBT sessions and/or maintenance pharmacotherapy.

We obtained estimates of health care resource use by depression status from the same study that provided the EQ-5D data (Kuyken et al., 2008) and again used a pooled ordinary least squares model with cluster robust standard errors in our estimation of these figures. We updated the costs from this study using the Hospital and Community Health Service Pay and Price Index (Curtis, 2012). To reflect the current value of the benefits and costs accumulating over the time horizon of the model we discounted both at a rate of $3.5 \%$ as suggested by NICE (2013). Table 1 summarises the model inputs that were not estimated in the meta-analyses.

\section{Cost-effectiveness and sensitivity analyses}

We calculated incremental cost-effectiveness ratios (ICER) by dividing the estimated mean differences in costs between two treatments by the mean difference in QALYs. To address uncertainty in the ICERs we undertook a probabilistic sensitivity analysis. This involved repeatedly simulating random draws from the distribution of the parameter inputs in order to determine the joint distribution of the outputs of the model (i.e. the relative mean cost and effects of the interventions). We displayed these distributions on a cost-effectiveness plane as credible ellipses. These regions indicate the 'true' cost-effectiveness estimates are likely to lie in and can thus be considered to be a two-dimensional generalisation of credible intervals.

Given the replications generated by the simulations, it was also possible to determine the net benefit of each intervention in each of the replications using the formula $N B=\lambda \cdot E-C$, where $C$ were the costs of the intervention, $E$ the benefits of the intervention in QALYs and $\lambda$ 
the value placed on a QALY by decision makers. We then determined the proportion of replications where each of the interventions had the highest net benefit (i.e. the probability that they were the most cost-effective). We displayed these data for a range of $\lambda$ using costeffectiveness acceptability curves (CEACs) (Fenwick and Byford, 2005). Values of the CEAC close to 1 or 0 indicated that the uncertainty as to whether the respective treatment was most likely to be the most cost-effective was low (Baio, 2012). In this study, we discuss the results for a range of $\lambda$ between $£ 20,000$ to $£ 30,000$ because this has been presumed to be the range of willingness to pay for QALY improvement by the NICE but we acknowledge that lower estimates have recently been suggested (Haycox, 2013).

We undertook four sensitivity analyses that had the potential to affect the results of the model. First, we considered the impact of relaxing the inclusion criteria for studies to be included in the meta-analysis of acute phase data. Unlike previous economic models, we focused exclusively on trials assessing CBT or CT. For that reason, amongst others, in our base case meta-analysis we excluded of a large trial by Keller et al. (2000), which assesses the efficacy of Cognitive Behavioural-Analysis System of Psychotherapy (CBASP). CBASP shares some of the features of CBT but differs from the way that it is commonly implemented due to its primary focus on interpersonal interactions (Driessen and Hollon, 2010). However, due to the size of this study, in our first sensitivity analysis we analysed the effect of including Keller et al. (2000). Second, the costing assumptions with respect to CBT in our base case scenario was likely to favour pharmacotherapy because typically patients tend to attend fewer appointments than set out in this treatment protocol even if completing treatment. In the studies included in the meta-analysis attendance rate was approximately $80 \%$. It was unclear what fraction of unattended session was cancelled without prior notice but to gain some understanding of the potential implications of non-attendance we reduced psychotherapy costs by $20 \%$ in our second sensitivity. Third, we relaxed the inclusion criteria for the follow-up period, incorporating studies in our meta-analysis in which drug treatment was provided after the end of the acute phase in the pharmacotherapy arm but not necessarily any maintenance treatment in the other two arms. Finally, we used SF-6D utility data from Kendrick et al. (2009) to assess the sensitivity of the estimated QALY gains to the choice of utility measure. As in the case of EQ-5D, we used a pooled ordinary least squares model with robust standard errors to estimate the mean utility scores. 


\section{Results}

We identified fifteen randomised trials that fulfilled our inclusion criteria for the acute phase. Eleven of these compared pharmacotherapy with CBT as a monotherapy, three compared pharmacotherapy with both CBT and combination treatment, and one compared CBT with combination treatment only. We report the raw data extracted from these studies in Appendix C. Figure 2 summarises the results of the meta-analysis at the end of the acute treatment phase and the meta-analyses at the two follow-ups. CBT and combination therapy both had higher proportion of patients remitting and a lower proportion of patients discontinuing prematurely than pharmacotherapy. Patients were more likely to complete CBT treatment than combination therapy, but the share of individuals whose depression status improved was similar. The probability of remission was higher under combination treatment than CBT. In the base case scenario, only three trials fulfilled our inclusion criteria for the follow-up phase, one of which included combination therapy. At 12 month follow-up the risk of relapse among remitters was estimated to be lowest for CBT (43\%), followed by combination therapy (49\%) and pharmacotherapy (55\%). The ranking of cumulative relapse rates was the same at 24 month follow-up with probabilities of $62 \%, 66 \%$ and $75 \%$ respectively (see Figure 2). Forest plots for all meta-analyses can be found in Appendix B.

The model suggested that pharmacotherapy and combination therapy had the lowest and highest expected costs respectively (£3645 vs. £5060). The estimate for CBT was $£ 4418$. QALYs were lowest in the pharmacotherapy arm (1.236), whereas those for CBT and combination treatment were identical up to a rounding error (1.274). Therefore, CBT yielded the same benefits as combination therapy while being less expensive, i.e. CBT dominated combination therapy. The ICER for CBT compared to pharmacotherapy was $£ 20,039 / Q A L Y$ (Table 2). Figure 3 shows CEACs comparing all three treatments at the same time. Within the aforementioned NICE threshold range there was much uncertainty. At the lower end, pharmacotherapy, the least expensive treatment, was most likely to be cost-effective, whereas at a threshold above around $£ 22,000$ per QALY this changes to CBT. There was considerable decision uncertainty because there was a strong chance of not choosing the most cost-effective treatment option when deciding on the treatment with the highest average net benefit, i.e. pharmacotherapy at a willingness to pay of less than $£ 20,039$ and CBT above that. Figure 3 also shows that combination treatment was least likely to be costeffective with the range of $£ 20,000$ to $£ 30,000$ per QALY. The estimated probability ranged from 15 to $23 \%$. 
Table 2 shows the results of the sensitivity analyses. As previously mentioned, to simplify comparison between the scenarios, we also display the estimated cost and effect differences for each of these graphically using cost-effectiveness planes in Appendix A. Including the study by Keller et al. (2000) in the meta-analysis substantially increased the costeffectiveness of combination treatment, such that it became the treatment most rather than least likely to be cost-effective. However, the decision uncertainty between the three treatment options remained high in this scenario. As indicated in Table 2 at a willingness to pay of $£ 25,000$ per QALY, the probabilities of pharmacotherapy (32\%), CBT (31\%) and combination treatment $(37 \%)$ being the most cost-effective treatment were comparable. As expected, reducing the CBT attendance rate in the second sensitivity analysis lowered the costs of both CBT monotherapy and combination treatment. This did not affect the ranking of treatments in terms of their cost-effectiveness but the ICER for CBT was considerably lower at $£ 9,714$ per QALY in this scenario. In the third sensitivity analysis, two additional studies were included in the meta-analysis of relapse rates. As a result, rather than CBT, relapse rates in were estimated to be lowest with combination therapy. However, the additional QALY gain of combination treatment over CBT was not sufficient to make it the treatment with the highest likelihood of being cost-effective and at $£ 61,403$ per QALY the ICER for combination therapy relative to CBT was high. Using SF-6D instead of EQ-5D to estimate QALYs decreased the absolute differences in QALYs between the treatments such that the ICER for CBT increased to $£ 32,582$ per QALY and at a willingness to pay for a QALY of $£ 25,000$ pharmacotherapy became the treatment most likely to be cost-effective $(51 \%$ probability). In summary, two sensitivity analyses affected the ranking of the interventions in terms of the probability of being the most cost-effective compared to our base case scenario and the ICERs for the interventions varied markedly between the scenarios under consideration.

\section{Discussion}

This study adds to the existing economic evaluations comparing psychological and pharmacological treatments for depression. Specifically, two decision models commissioned by NICE exist examining a comparable decision problem as our study. However, these analyses come to different conclusions than our appraisal of the evidence. NICE (2009) reported ICERs of $£ 7,052$ and $£ 5,558$ per QALY respectively for combination therapy relative to pharmacotherapy in the treatment of moderate and severe depression in secondary care. In the related study Simon et al. (2006) the corresponding ICERs were 
$£ 4,056$ and $£ 14,540$ per QALY. By contrast, our analysis suggested that CBT as monotherapy was more likely to be cost-effective than pharmacotherapy which in turn was more likely to be cost-effective than combination treatment. In addition, the decision uncertainty was much greater in our model, i.e. the difference in the probability of being costeffective between the treatments as suggested by the CEACs was lower.

These discrepancies are largely due to four differences in the modelling approach. First, we specified different inclusion criteria than these earlier studies. For example, we excluded data by Simons et al. (1986) which suggested a large difference in relapse rates between pharmacotherapy and combination therapy because, contrary to NICE guidance, treatment was discontinued in this study following the end of the acute phase. Also, we restricted our analysis to studies on CBT or CT rather than allowing for all types of psychotherapies. In our sensitivity analysis we showed that excluding the trial by Keller et al. (2000) which reported large benefits of combination treatment over the two monotherapies for a type of psychological therapy that is not commonly provided in the NHS has a significant effect on the cost-effectiveness estimate of combination treatment. Second, we used more modern methods of evidence synthesis. As a result, for example, when comparing the effect of pharmacotherapy and combination therapy on remission rates, the network meta-analysis estimates are smaller than those in a traditional pairwise analysis because it accounts for the fact that in trials including all three treatments, the treatment effect for CBT was above average compared to the trials which did not include combination treatment. In statistical terms, it accounted for trial arms not missing completely at random. Third, the time horizon of the study was extended beyond 15-month. This particularly improved the costeffectiveness of CBT since, at least in the base case scenario, the meta-analysis suggested that was superior to the other two treatments in preventing relapse. Fourth, our model was based on different health states and disease trajectories. For example, we differentiated between patients showing full and partial response rather than implicitly assuming that all patients who did not remit showed no response. On the other hand, we considered the information reported in clinical trials to be too limited to differentiate between the treatment effects for patients with moderate depression and those with severe depression at baseline. Instead, we modelled the cost-effectiveness for these two populations as a whole.

Besides these differences in the cost-effectiveness estimates, we would also argue that the conclusions that can be drawn from the results of our economic model and its predecessors are more limited than implied by NICE (2009) guidelines. Current recommendations do not differentiate between treatment in a primary and secondary care setting but the vast majority of the data used to inform the parameters in this model as well as the analyses by NICE (2009) and Simon et al. (2006) draw from the clinical literature set in secondary care. One 
would expect depression severity and general health status of patients to be worse in this setting and there are also likely to be different implications for resource use than in primary care. Therefore, we would argue that the conclusions drawn from this model cannot easily be transferred to treatment of depression in primary care, the setting in which most patients with depression are cared for in the UK. The differences between the results of this model and its predecessors do, however, illustrate the fragility of cost-effectiveness estimates in the depression. Given investments of over $£ 400$ million to increase the availability of psychological treatments in primary care as part of the improving access to psychological therapies (IAPT) initiative, this also highlights the need for further examination of the economic evidence for treatment of depression in UK primary care (Clark, 2011, Department of Health, 2012).

We invite the reader to critically examine our choice of inputs, synthesis method, model structure and the validity of the conclusions drawn in light of the available evidence (Afzali et al., 2012a, b). Decision models can be useful in synthesising, linking and extrapolating a wide variety of information in a transparent fashion, but they are inevitably reductionist and can only be regarded as decision aids (something that could be also argued to apply to RCTs). In the appraisal of the evidence base, modellers are faced with an inevitable tradeoff between competing objectives such as precision, relevance, validity and feasibility. This model is no exception from most other health economic models in that it is heavily dependent on structural assumptions, i.e. it largely omits non-statistical uncertainty (Grutters et al., 2014).

Our meta-analyses combined heterogeneous treatments to yield a single treatment effect estimate. Thus, rather than taking the 'best' antidepressant as the relevant benchmark, as was the case in NICE (2009) and Simon et al. (2006), we evaluated pharmacotherapy and combination therapy as a class which may have biased results to some extent. Similarly, both in psychological therapy and pharmacotherapy, variations in non-specific elements, treatment fidelity and the therapeutic relationship across studies are likely to have some bearing on treatment effectiveness which may have been a source of bias. However, there was a dearth of evidence with respect to some model parameters, particularly response and relapse rates in combination therapy. Therefore, we believe that our approach was preferable to applying of further quality criteria as this would have further reduced the robustness of the model or required additional subjective judgements. Unlike previous studies, we consistently used the HAM-D as the backbone of the model to enhance the internal consistency of the model, however, to do so it was necessary to derive key model parameter estimates from a single trial carried out in a primary care setting (Kuyken et al., 2008). Since patients in primary care are likely to consume less health care resources 
beyond the resources used for depression treatment, this is likely to have underestimate the cost-effectiveness of the more effective treatments, i.e. combination treatment and CBT, to some extent. Furthermore, the psychometric properties of HAM-D have been debated and may have biased our comparison (Fountoulakis et al., 2014). This study applies more modern statistical methods to estimate parameters and account for uncertainty than its predecessors, but, amongst others, a limitation which remains in our meta-analysis is the assumption that there is no correlation in outcomes within treatment arms which is unlikely to hold.

Another feature of our model that should be emphasised is that, like its precursors, the efficacy of the interventions was based primarily on RCT data given its status in the NICE hierarchy of evidence and because adequate observational data was not available. Yet, this also represents a limitation of this study because the generalisability of RCTs in depression to routine clinical practice has been questioned (van der Lem et al., 2012). Since reporting on side effects, suicide risks and the exact timing of events in these trials is inconsistent or incomplete, this also meant that we did not incorporate these into the decision model. The exclusion of adverse drug reactions evidently favoured pharmacotherapy and combination therapy but there does not appear to be clear evidence to indicate what the direction of bias in our model might be because of the omission of other two factors. In addition, in all relevant RCTs antidepressants were tapered off after 6 or 12 months follow-up for all patients in the pharmacotherapy and combination treatment arm. However, due to increased risk of relapse after withdrawal from medications, NICE (2009) guidelines advise continuation of antidepressant treatments for longer than that for at risk patients which is increasingly the case in routine clinical practice (Moore et al., 2009). It is unclear what effect this discrepancy between naturalistic and trial prescribing practice might have on the relative cost-effectiveness of pharmacotherapy and combination treatment. We would also like to emphasise that, at best, data from RCTs only applies to individuals who are likely to participate in them. Some patients treated for depression in secondary care are likely to be so unwell that they are unlikely to be enrolled in a typical trial and in practice many patients with MDD have strong preferences for pharmacotherapy or psychotherapy which should be taken into account in the choice of treatment for depression (NICE, 2009, van Schaik et al., 2004). 


\section{Conclusion}

In this paper we have revisited the evidence base for treatment of depression in UK secondary care. Our economic appraisal differs from the recommendation made by current NICE (2009) guidance and our decision model may therefore be of use when reviewing treatment recommendations for depression in the future. In a routine care setting, local consideration and the constraints in the supply of CBT are likely to play a role in treatment decisions, but this model may offer a platform for further discussion of the cost-effectiveness of interventions (Gyani et al., 2012). We found the evidence base comparing pharmacotherapy, CBT and combination therapy for depression to be remarkably limited, particularly with respect to the latter. Thus, besides further economic evaluations in UK primary care, we would like to suggest three areas of uncertainty that, we believe, would particularly warrant more in depth exploration. First, the relative cost-effectiveness of different types of interventions for depression is likely to differ between subgroup of patients. Personalising the choice of treatment will be critical to more efficient use of health care resources in the future (Cuijpers et al., 2012, Hollinghurst et al., 2014, Simon and Perlis, 2010, Wallace et al., 2013). Second, we would encourage the use of observational data coupled with appropriate statistical methods to gain a better understanding of the effects of depression treatment in a routine care setting and the long-term cost-effectiveness. Third, the wider societal impact of depression treatments and the way that it should be incorporated into health technology assessment is currently unclear due to a host of methodological, ethical, policy and practical issues (NICE, 2014, Sculpher, 2001). However, recent evidence suggests that CBT may produce greater improvements in employability which warrants further exploration (Fournier et al., 2014).

\section{Acknowledgements}

We would like to thank Sarah Byford and Barbara Barrett for allowing us to make use of some of their data for this study. We also thank participants of the ENMESH 2013 conference, Petr Winkler and particularly three anonymous reviewers for their valuable comments on earlier versions of this study.

\section{Conflicts of interest}

None 


\section{References}

Afzali, H. H. A., Karnon, J. \& Gray, J. (2012a). A Critical Review of Model-Based Economic Studies of Depression. PharmacoEconomics 30, 461-482.

Afzali, H. H. A., Karnon, J. \& Gray, J. (2012b). A proposed model for economic evaluations of major depressive disorder. The European Journal of Health Economics 13, 501-510.

American Psychiatric Association (2000). Handbook of psychiatric measures. Amer Psychiatric Pub Incorporated.

Baio, G. (2012). Bayesian Methods in Health Economics. CRC Press.

Borenstein, M., Hedges, L. V., Higgins, J. P. T. \& Rothstein, H. R. (2009). Introduction to MetaAnalysis. Wiley.

Clark, D. M. (2011). Implementing NICE guidelines for the psychological treatment of depression and anxiety disorders: The IAPT experience. International Review of Psychiatry 23, 318-327.

Cuijpers, P., Reynolds, C. F., Donker, T., Li, J., Andersson, G. \& Beekman, A. (2012). Personalized treatment of adult depression: medication, psychotherapy, or both? A systematic review. Depression and Anxiety 29, 855-864.

Cuijpers, P., van Straten, A., Warmerdam, L. \& Andersson, G. (2008). Psychological treatment of depression: A meta-analytic database of randomized studies. BMC Psychiatry 8, 36.

Curtis, L. (2012). Unit Costs of Health and Social Care 2012. Personal Social Services Research Unit. Department of Health (2012). IAPT three-year report - The first million patients. London.

Dias, S., Sutton, A. J., Ades, A. E. \& Welton, N. J. (2013a). Evidence Synthesis for Decision Making 2: A Generalized Linear Modeling Framework for Pairwise and Network Meta-analysis of Randomized Controlled Trials. Medical Decision Making 33, 607-617.

Dias, S., Sutton, A. J., Welton, N. J. \& Ades, A. E. (2013b). Evidence Synthesis for Decision Making 3: Heterogeneity-Subgroups, Meta-Regression, Bias, and Bias-Adjustment. Medical Decision Making 33, 618-640.

Dias, S., Welton, N. J., Sutton, A. J. \& Ades, A. E. (2013c). Evidence Synthesis for Decision Making 5: The Baseline Natural History Model. Medical Decision Making 33, 657-670.

Driessen, E. \& Hollon, S. D. (2010). Cognitive behavioral therapy for mood disorders: efficacy, moderators and mediators. Psychiatr Clin North Am 33, 537-55.

Fenwick, E. \& Byford, S. (2005). A guide to cost-effectiveness acceptability curves. The British Journal of Psychiatry 187, 106-108.

Fountoulakis, K. N., Samara, M. T. \& Siamouli, M. (2014). Burning issues in the meta-analysis of pharmaceutical trials for depression. Journal of Psychopharmacology 28, 106-117.

Fournier, J. C., DeRubeis, R. J., Amsterdam, J., Shelton, R. C. \& Hollon, S. D. (2014). Gains in employment status following antidepressant medication or cognitive therapy for depression. The British Journal of Psychiatry.

Gelman, A., Jakulin, A., Pittau, M. G. \& Su, Y.-S. (2008). A weakly informative default prior distribution for logistic and other regression models. 1360-1383.

Grutters, J. C., van Asselt, M. A., Chalkidou, K. \& Joore, M. (2014). Healthy Decisions: Towards Uncertainty Tolerance in Healthcare Policy. PharmacoEconomics, 1-4.

Gyani, A., Pumphrey, N., Parker, H., Shafran, R. \& Rose, S. (2012). Investigating the use of NICE guidelines and IAPT services in the treatment of depression. Mental Health in Family Medicine $\mathbf{9}$, 149-160.

Haycox, A. (2013). How Much Should the NHS Pay for a QALY? PharmacoEconomics 31, 357-359. Higgins, J. P. \& Green, S. (2008). Cochrane handbook for systematic reviews of interventions. Wiley Online Library.

Hollinghurst, S., Carroll, F. E., Abel, A., Campbell, J., Garland, A., Jerrom, B., Kessler, D., Kuyken, W., Morrison, J., Ridgway, N., Thomas, L., Turner, K., Williams, C., Peters, T. J., Lewis, G. \& Wiles, N. (2014). Cost-effectiveness of cognitive-behavioural therapy as an adjunct to pharmacotherapy for 
treatment-resistant depression in primary care: economic evaluation of the CoBalT Trial. $B r J$ Psychiatry 204, 69-76.

llyas, S. \& Moncrieff, J. (2012). Trends in prescriptions and costs of drugs for mental disorders in England, 1998-2010. The British Journal of Psychiatry 200, 393-398.

Jarrett, R. B., Kraft, D., Schaffer, M., Witt-Browder, A., Risser, R., Atkins, D. H. \& Doyle, J. (2000). Reducing relapse in depressed outpatients with atypical features: a pilot study. Psychother Psychosom 69, 232-9.

Keller, M. B., McCullough, J. P., Klein, D. N., Arnow, B., Dunner, D. L., Gelenberg, A. J., Markowitz, J. C., Nemeroff, C. B., Russell, J. M., Thase, M. E., Trivedi, M. H., Blalock, J. A., Borian, F. E., Jody, D. N., DeBattista, C., Koran, L. M., Schatzberg, A. F., Fawcett, J., Hirschfeld, R. M. A., Keitner, G., Miller, I., Kocsis, J. H., Kornstein, S. G., Manber, R., Ninan, P. T., Rothbaum, B., Rush, A. J., Vivian, D. \& Zajecka, J. (2000). A Comparison of Nefazodone, the Cognitive Behavioral-Analysis System of Psychotherapy, and Their Combination for the Treatment of Chronic Depression. New England Journal of Medicine 342, 1462-1470.

Kendrick, T., Chatwin, J., Dowrick, C., Tylee, A., Morriss, R., Peveler, R., Leese, M., McCrone, P., Harris, T., Moore, M., Byng, R., Brown, G., Barthel, S., Mander, H., Ring, A., Kelly, V., Wallace, V., Gabbay, M., Craig, T. \& Mann, A. (2009). Randomised controlled trial to determine the clinical effectiveness and cost-effectiveness of selective serotonin reuptake inhibitors plus supportive care, versus supportive care alone, for mild to moderate depression with somatic symptoms in primary care: the THREAD (THREshold for AntiDepressant response) study. Health Technol Assess 13, iii-iv, ixxi, 1-159.

Kuyken, W., Byford, S., Taylor, R. S., Watkins, E., Holden, E., White, K., Barrett, B., Byng, R., Evans, A., Mullan, E. \& Teasdale, J. D. (2008). Mindfulness-based cognitive therapy to prevent relapse in recurrent depression. J Consult Clin Psychol 76, 966-78.

Malek, M. H. (2000). Implementing QALYs. Hayward Medical Communications.

McCrone, P., Dhanasiri, S., Patel, A., Knapp, M. \& Lawton-Smith, S. (2008). Paying the price: the cost of mental health care in England to 2026. King's Fund: London.

Moore, M., Yuen, H. M., Dunn, N., Mullee, M. A., Maskell, J. \& Kendrick, T. (2009). Explaining the rise in antidepressant prescribing: a descriptive study using the general practice research database. NICE (2009). Depression: The NICE guideline on the treatment and management of depression updated edition. In National Clinical Practice Guideline 90 (ed. National Collaborating Centre for Mental Health). The British Psychological Society and The Royal College of Psychiatrists.

NICE (2013). Guide to the methods of technology appraisal 2013. National Institute for Health and Care Excellence,: London.

NICE (2014). Value Based Assessment of Health Technologies. (ed. N. I. F. H. A. C. EXCELLENCE). Radhakrishnan, M., Hammond, G., Jones, P. B., Watson, A., McMillan-Shields, F. \& Lafortune, L. (2013). Cost of Improving Access to Psychological Therapies (IAPT) programme: An analysis of cost of session, treatment and recovery in selected Primary Care Trusts in the East of England region.

Behaviour Research and Therapy 51, 37-45.

Riley, R. D., Higgins, J. P. T. \& Deeks, J. J. (2011). Interpretation of random effects meta-analyses. BMJ 342.

Sado, M., Knapp, M., Yamauchi, K., Fujisawa, D., So, M., Nakagawa, A., Kikuchi, T. \& Ono, Y. (2009). Cost-effectiveness of combination therapy versus antidepressant therapy for management of depression in Japan. Australian and New Zealand Journal of Psychiatry 43, 539-547.

Sculpher, M. (2001). The role and estimation of productivity costs in economic evaluation. Economic evaluation in health care: merging theory with practice, 94-112.

Simon, G. E. \& Perlis, R. H. (2010). Personalized medicine for depression: can we match patients with treatments? Am J Psychiatry 167, 1445-55.

Simon, J., Pilling, S., Burbeck, R. \& Goldberg, D. (2006). Treatment options in moderate and severe depression: decision analysis supporting a clinical guideline. The British Journal of Psychiatry 189, 494-501. 
Simons, A. D., Murphy, G. E., Levine, J. L. \& Wetzel, R. D. (1986). Cognitive therapy and pharmacotherapy for depression. Sustained improvement over one year. Arch Gen Psychiatry 43, 438.

Turner, R. M., Davey, J., Clarke, M. J., Thompson, S. G. \& Higgins, J. P. (2012). Predicting the extent of heterogeneity in meta-analysis, using empirical data from the Cochrane Database of Systematic Reviews. Int J Epidemiol 41, 818-27.

van der Lem, R., van der Wee, N. J. A., van Veen, T. \& Zitman, F. G. (2012). Efficacy versus Effectiveness: A Direct Comparison of the Outcome of Treatment for Mild to Moderate Depression in Randomized Controlled Trials and Daily Practice. Psychotherapy and Psychosomatics 81, 226-234. van Schaik, D. J. F., Klijn, A. F. J., van Hout, H. P. J., van Marwijk, H. W. J., Beekman, A. T. F., de Haan, M. \& van Dyck, R. (2004). Patients' preferences in the treatment of depressive disorder in primary care. General Hospital Psychiatry 26, 184-189.

Wallace, M. L., Frank, E. \& Kraemer, H. C. (2013). A novel approach for developing and interpreting treatment moderator profiles in randomized clinical trials. JAMA Psychiatry 70, 1241-1247. 